\title{
Investigating cellular network heterogeneity and modularity in cancer: a network entropy and unbalanced motif approach
}

Feixiong Cheng ${ }^{1 \dagger}$, Chuang $\mathrm{Liu}^{2+}$, Bairong Shen ${ }^{3}$ and Zhongming Zhao ${ }^{1,4,5,6^{*}}$

From The International Conference on Intelligent Biology and Medicine (ICIBM) 2015

Indianapolis, IN, USA. 13-15 November 2015

\begin{abstract}
Background: Cancer is increasingly recognized as a cellular system phenomenon that is attributed to the accumulation of genetic or epigenetic alterations leading to the perturbation of the molecular network architecture. Elucidation of network properties that can characterize tumor initiation and progression, or pinpoint the molecular targets related to the drug sensitivity or resistance, is therefore of critical importance for providing systems-level insights into tumorigenesis and clinical outcome in the molecularly targeted cancer therapy.

Results: In this study, we developed a network-based framework to quantitatively examine cellular network heterogeneity and modularity in cancer. Specifically, we constructed gene co-expressed protein interaction networks derived from large-scale RNA-Seq data across 8 cancer types generated in The Cancer Genome Atlas (TCGA) project. We performed gene network entropy and balanced versus unbalanced motif analysis to investigate cellular network heterogeneity and modularity in tumor versus normal tissues, different stages of progression, and drug resistant versus sensitive cancer cell lines. We found that tumorigenesis could be characterized by a significant increase of gene network entropy in all of the 8 cancer types. The ratio of the balanced motifs in normal tissues is higher than that of tumors, while the ratio of unbalanced motifs in tumors is higher than that of normal tissues in all of the 8 cancer types. Furthermore, we showed that network entropy could be used to characterize tumor progression and anticancer drug responses. For example, we found that kinase inhibitor resistant cancer cell lines had higher entropy compared to that of sensitive cell lines using the integrative analysis of microarray gene expression and drug pharmacological data collected from the Genomics of Drug Sensitivity in Cancer database. In addition, we provided potential network-level evidence that smoking might increase cancer cellular network heterogeneity and further contribute to tyrosine kinase inhibitor (e.g., gefitinib) resistance.

(Continued on next page)
\end{abstract}

\footnotetext{
* Correspondence: zhongming.zhao@uth.tmc.edu

${ }^{\dagger}$ Equal contributors

${ }^{1}$ Department of Biomedical Informatics, Vanderbilt University School of

Medicine, Nashville, TN, USA

${ }^{4}$ Department of Cancer Biology, Vanderbilt University School of Medicine,

Nashville, TN, USA

Full list of author information is available at the end of the article
} 
(Continued from previous page)

Conclusion: In summary, we demonstrated that network properties such as network entropy and unbalanced motifs associated with tumor initiation, progression, and anticancer drug responses, suggesting new potential network-based prognostic and predictive measure in cancer.

Keywords: Cancer, Heterogeneity, Network modularity, Network entropy, Unbalanced motifs

Abbreviations: CePIN, Co-expressed Protein Interaction Network; ICGC, International Cancer Genome Consortium; OG, Oncogene; PCC, Pearson Correlation Coefficient; PIN, Protein Interaction Network; PPI, Protein-Protein Interaction; TCGA, The Cancer Genome Atlas; TSG, Tumor Suppressor Gene

\section{Background}

Cancer is a major public health problem in the world and approximately $25 \%$ of deaths in the United States is due to cancer [1]. Analyses of massive amounts of cancer genomics data generated from The Cancer Genome Atlas (TCGA) and the International Cancer Genome Consortium (ICGC) has suggested that cancer is a systems-level, network phenomenon attributed to the accumulation of genetic or epigenetic alterations under molecular network architecture [2-4]. However, our understanding of cancer biology at the systems-level has still been nascent, such as genome stability or instability $[5,6]$. There is an urgent need to develop network-based methods or approaches to explore systems-level, network features associated with tumor initiation, progression, and resistance of specific targeted agents so that such findings will provide new potential prognostic and therapeutic biomarkers in cancer.

Several network terms, such as "cancer network attractors" [7], "network plasticity" [8], and "network entropy" [9-12], had been proposed in cancer systems biology study. West et al. found that cancer cells often have higher network entropy by integrating microarray gene expression data into a protein interaction network (PIN) [9]. Banerji et al. suggested that signaling entropy provided a potential measure in cancer by investigating microarray gene expression data in 3668 breast cancer samples and 1692 lung adenocarcinoma samples [10]. Therefore, network entropy can be a useful quantitative measure to characterize different disease status, like tumor versus normal tissue as well as various stages of progression.

In molecularly targeted cancer therapeutics, the most common approach is to find molecules that can directly lead to the death of cancer cells, such as kinase inhibitors. However, targeted agents (e.g., kinase inhibitors) often develop high risk of drug resistance due to the feedback or crosstalk signaling mechanisms within cellular networks [13]. One possible reason is that currently targeted therapy often introduces stress and further lead to increase the degree of heterogeneity of a cancer cell population in the long therapeutic period despite shortterm induction of cancer cell death [14]. The end result will be to speedup the process of drug resistance through cancer evolution. Furthermore, normal cells will be at a survival disadvantage as they are much less dynamic than cancer cells. However, our understanding of the systems-level network features that characterize anticancer drug responses has been largely behind the clinical practice in cancer fields.

In this study, we proposed an integrated networkbased framework to examine whether network properties (e.g. network entropy or unbalanced motifs) are associated with tumor initiation and progression, and anticancer drug responses (Fig. 1). Specifically, we built each co-expressed PIN (CePIN) to describe specific cellular network statuses characterizing tumorigenesis, progression (four stages), and anticancer drug responses by integrating the large-scale RNA-Seq data across 8 cancer types from TCGA and the microarray gene expression data from the Genomics of Drug Sensitivity in Cancer (GDSC) database $[15,16]$. We then employed network entropy and balanced versus unbalanced motif approaches to quantitatively characterize tumor initiation, progression, and anticancer drug responses. Using network entropy as measure, we found that cells could be characterized by an increase in both network entropy and unbalanced motifs during tumorigenesis. Moreover, we could use the network entropy to quantify various stages of progression and anticancer drug responses. In summary, this study would provide new potential network-based prognostic and predictive biomarkers in cancer.

\section{Results}

\section{Overview of a network entropy and unbalanced motif approach}

In this study, we proposed a network-based entropy and unbalanced motif approach based on the notion of Waddington's landscape $[10,17]$ as shown in Fig. 1. First, we built each gene CePIN by incorporating RNA-Seq V2 data covering tumor and normal tissue samples across 8 cancer types from TCGA into a high-quality PIN (Fig. 1a). These 8 cancer types consist of breast invasive carcinoma (BRCA), colon adenocarcinoma (COAD), head and neck squamous cell carcinoma (HNSC), kidney 


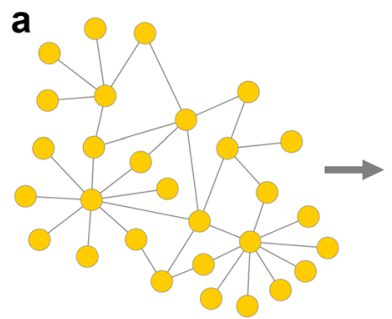

Protein protein interaction (PPI)

b

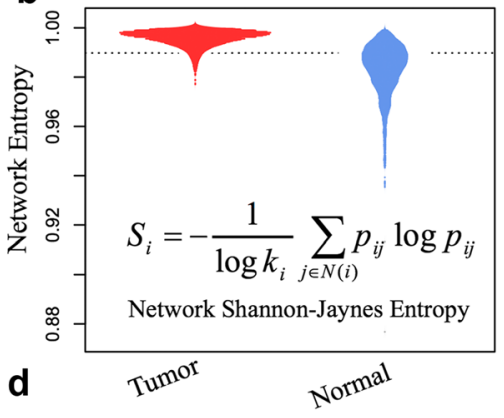

Normal samples Tumor samples

$\mathrm{P}_{1} \mathrm{P}_{2} \cdots \mathrm{P}_{\mathrm{n}} \mathrm{P}^{\prime}{ }_{1} \mathrm{P}_{2} \cdots \mathrm{P}^{\prime}$

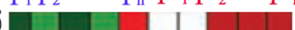

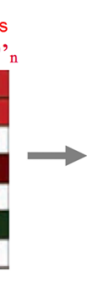

Gene transcriptome
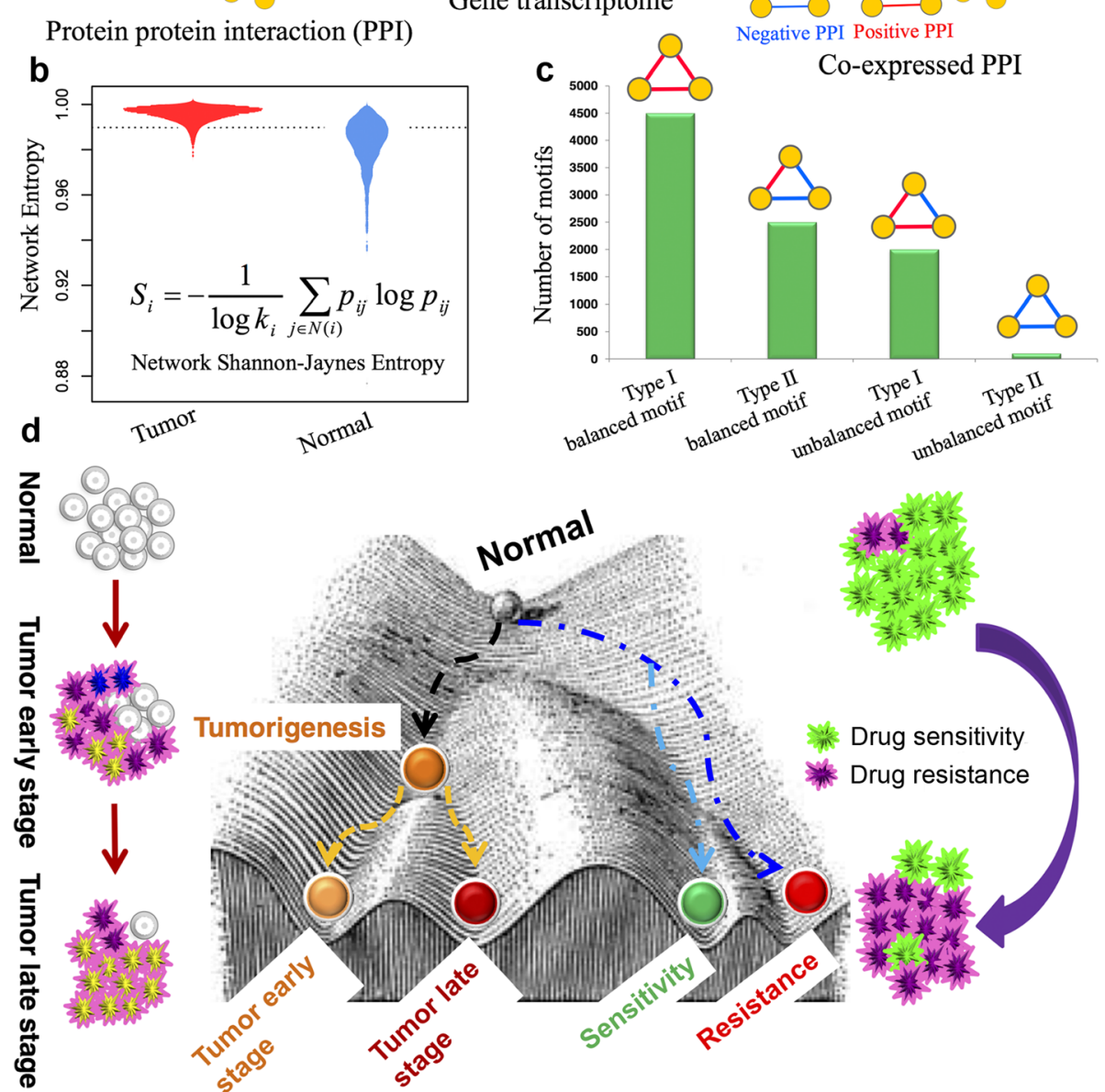

Drug sensitivity

Drug resistance

Fig. 1 Diagram of the network entropy and unbalanced motif approach to quantify cancer cellular network heterogeneity and modularity. a Workflow for gene co-expressed protein interaction network construction using large-scale RNA-Seq data from The Cancer Genome Atlas. Network entropy (b), and $\mathbf{c}$ balanced versus unbalanced motif analysis. $\mathbf{d}$ Quantifying cancer cellular network heterogeneity and modularity characterized by network entropy, and balanced versus unbalanced motifs in tumor versus matched normal samples, different stages of tumor progression, and anticancer drug sensitive versus resistant cancer cell lines. Based on the notion of Waddington's landscape [17], network entropy and ratio of unbalanced versus balanced motifs were represented as the energy potential in Waddington's landscape. High cellular network heterogeneity during tumorigenesis is characterized by elevated network entropy and high ratio of unbalanced versus balanced motifs

renal clear cell carcinoma (KIRC), lung adenocarcinoma (LUAD), lung squamous cell carcinoma (LUSC), thyroid carcinoma (THCA), and uterine corpus endometrial carcinoma (UCEC). We next built each CePIN to characterize anticancer drug sensitive versus resistant cancer cell lines using microarray gene expression and drug pharmacological data from GDSC database $[15,16]$. We then performed network analysis, such as network entropy and balanced versus unbalanced motifs in each CePIN (Fig. 1b and c). Finally, we systematically examined cellular network heterogeneity and modularity through aforementioned network measures in tumors versus normal tissue samples, various stages of tumor progression, and anticancer drug resistant versus sensitive cancer cell lines, respectively (Fig. 1d). Based on the notion of Waddington's landscape (Fig. 1d), we used network entropy and a ratio of unbalanced versus balanced motifs as the energy potential in Waddington's landscape $[10,17]$. Thus, we speculated that network entropy and the ratio of unbalanced versus balanced motifs can be used to quantitatively 
characterize cellular network heterogeneity and modularity in cancer. For instance, highly cellular network heterogeneity during tumorigenesis is marked by high network entropy and high ratio of unbalanced versus balanced motifs.

\section{Increased cellular network entropy during tumorigenesis}

We collected and processed the RNA-Seq V2 data (Additional file 1: Table S1) for tumor and normal tissue samples in 8 cancer types from TCGA, since only these 8 cancer types had at least 10 tumor samples and 10 normal samples. A good number of samples in gene expression data are required to build the reliable CePIN and to perform the follow up analyses. We calculated Pearson Correlation Coefficient (PCC) using RNA-Seq V2 data for each cancer type and available normal tissue samples, and then mapped PCC to large-scale PIN to build CePIN (Fig. 1a). Each CePIN contains $\sim 100,000$ edges and $\sim 10,000$ genes. We then calculated network entropy for each gene node in CePIN based on a previous study [9] (see Materials and Methods). We first examined genomewide $(\sim 10,000$ genes) local network entropy between tumor and normal tissues. As shown in Fig. 2, we found that tumors had higher genome-wide local network entropy compared to that of normal tissues in all of the 8 cancer types $\left(p<2.2 \times 10^{-16}\right.$, Wilcoxon rank-sum test). There was minor variation of network entropy for each gene as shown in Fig. 2, consistent with several previous studies [9-11].

We next examined genome-wide local network entropy distribution for 8 normal tissue types and 8 cancer types, respectively. Among the 8 normal tissues, colon had the lowest average genome-wide local network entropy $(0.9670 \pm 0.0003)$, while lung squamous had the highest average genome-wide local network entropy $(0.9822 \pm 0.0002)$, as shown in Fig. 2 . The observation of different local network entropy in different normal tissues might be due to tissue differentiation during development $[10,18]$. However, Fig. 2 showed that different cancer types had heterogeneous genome-wide local network entropy distribution compared to that of 8 normal tissues. Among the 8 cancer types, four: LUSC $(0.9957 \pm$ 0.0001), LUAD (0.9950 \pm 0.0001$), \quad$ BRCA $(0.9947 \pm$ $0.0001)$, and HNSC $(0.9945 \pm 0.0001)$, showed the highest average local network entropy distribution. The observation of high local network entropy in breast cancer might be explained by its high tumor heterogeneity [19]. For LUAD, LUSC, and HNSC, some environmental factors, such as smoking, may promote tumor heterogeneity and accordingly, cause higher network entropy. A previous study revealed that an average somatic mutation frequency in smokers was more than 10-fold higher in never-smokers in non-small cell lung cancer [20]. To test this hypothesis, we further separated TCGA patients
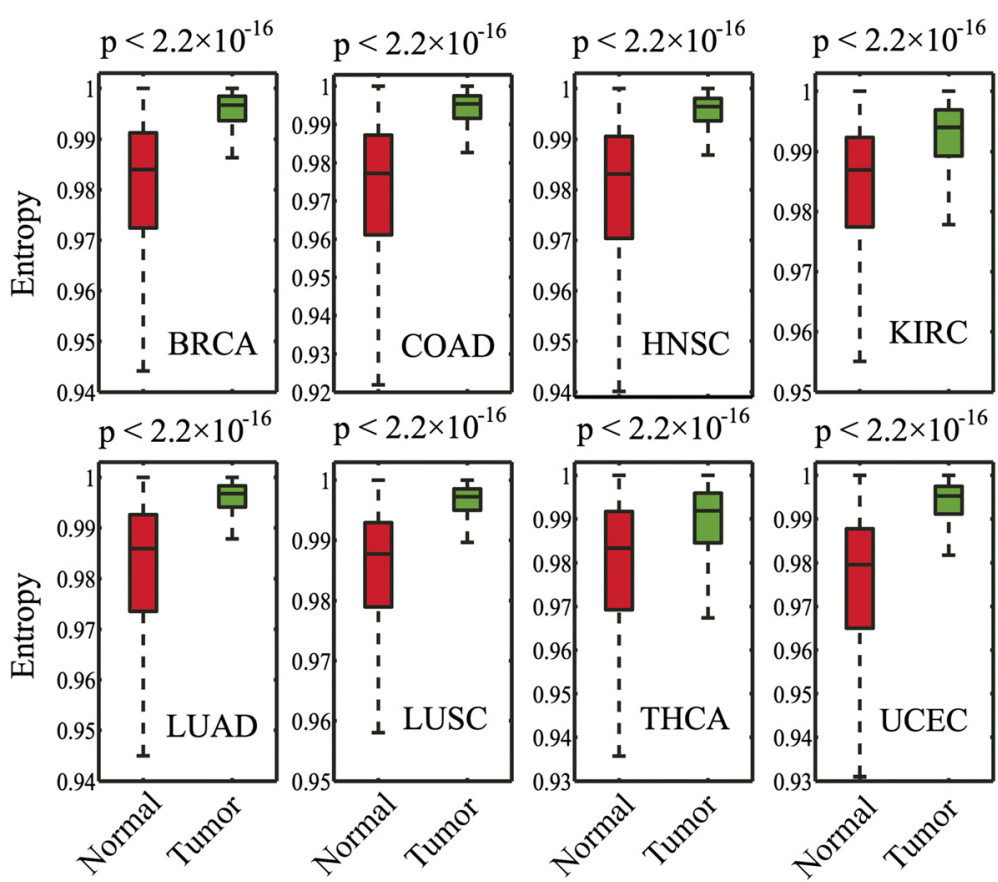

Fig. 2 Boxplots showing the distribution of genome-wide ( 10,000 genes) local network entropy between tumor samples and normal tissue samples for 8 cancer types. Breast invasive carcinoma (BRCA), colon adenocarcinoma (COAD), head and neck squamous cell carcinoma (HNSC), kidney renal clear cell carcinoma (KIRC), lung adenocarcinoma (LUAD), lung squamous cell carcinoma (LUSC), thyroid carcinoma (THCA), and uterine corpus endometrial carcinoma (UCEC). The p-value was calculated by Wilcoxon rank-sum test 


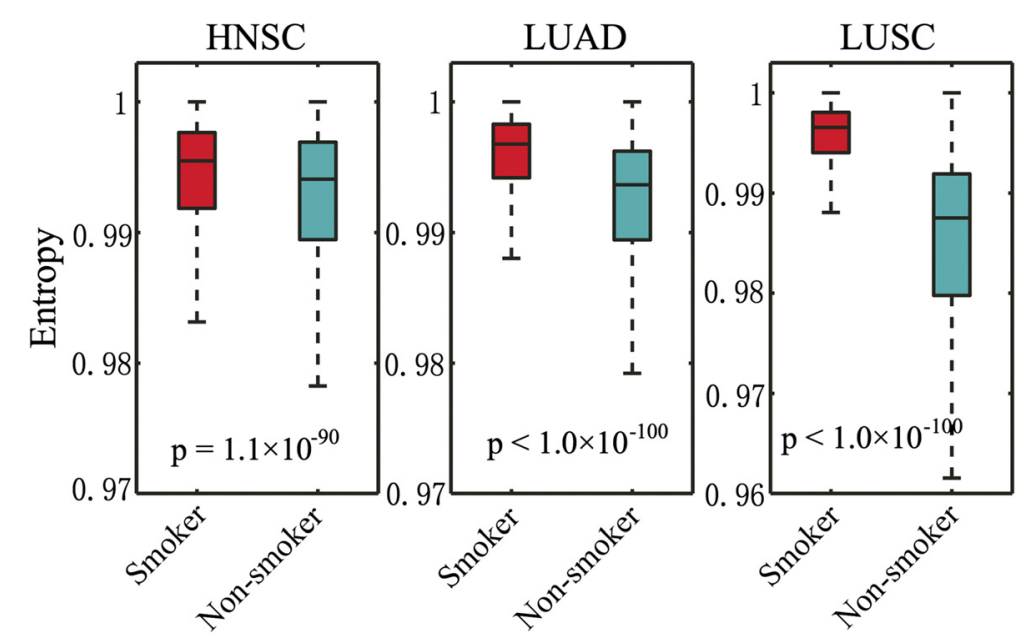

Fig. 3 Boxplots showing the distribution of genome-wide local network entropy between smokers and non-smokers in 3 cancer types. Lung adenocarcinoma (LUAD), lung squamous cell carcinoma (LUSC), and head and neck squamous cell carcinoma (HNSC). The p-value was calculated by Wilcoxon rank-sum test

into smokers and never-smokers in LUAD, LUSC, and HNSC, and rechecked the genome-wide local network entropy distribution. Figure 3 shows that smokers are characterized by a higher network entropy compared to that of non-smokers in all of the 3 smoking-related cancer types: LUAD $\left(p<1.0 \times 10^{-100}\right)$, LUSC $\left(p<1.0 \times 10^{-100}\right)$, and HNSC $\left(p=1.1 \times 10^{-90}\right)$.

\section{An increase of cellular network unbalanced motifs during tumorigenesis}

We next studied the network structural balance theory using our data. Specifically, we examined the ratio of unbalanced versus balanced motifs to quantify the stability of a network structure in a given condition (e.g., tumors versus normal tissues) $[21,22]$. As shown in Table 1, similar to social networks, normal tissues had more balanced motifs than that of tumors. Importantly, tumors had more unbalanced motifs than that of normal tissues in all of the 8 cancer types we examined. Unbalanced

Table 1 Distribution of balanced versus unbalanced motifs in tumor samples and normal tissues in 8 cancer types

\begin{tabular}{lccccc}
\hline \multirow{2}{*}{$\begin{array}{l}\text { Cancer } \\
\text { type }\end{array}$} & \multicolumn{2}{c}{ Fraction of balanced motifs } & & \multicolumn{2}{l}{ Fraction of unbalanced motifs } \\
\cline { 2 - 3 } & Normal & Tumor & & Normal & Tumor \\
\hline BRCA & 0.913 & 0.835 & & 0.087 & 0.165 \\
COAD & 1.00 & 0.919 & & 0.000 & 0.081 \\
HNSC & 0.994 & 0.909 & & 0.006 & 0.091 \\
KIRC & 0.948 & 0.819 & & 0.052 & 0.181 \\
LUAD & 0.979 & 0.879 & & 0.021 & 0.121 \\
LUSC & 0.979 & 0.877 & & 0.021 & 0.123 \\
THCA & 0.984 & 0.845 & & 0.016 & 0.155 \\
UCEC & 1.00 & 0.980 & & 0.000 & 0.020 \\
\hline
\end{tabular}

motifs are particularly interesting because they are highly dynamic and unstable [23]. For example, type II unbalanced motifs (Fig. 1c), consisting of two positive and one negative gene-gene interactions, should potentially be recognized as negative feedback loops or incoherent feed-forward loops. These two kinds of loops are both associated with adaptation responses and may be crucial for tumor cellular network system controllability.

\section{Characterizing tumor progression by network entropy}

We next investigated whether network entropy is associated with different stages of tumor progression. We collected the available RNA-Seq V2 data across 4 stages (I to IV) in 6 cancer types from TCGA, since only 6 cancer types had sufficient number ( $>10$ samples) of samples in each stage for building gene CePIN (Additional file 1: Table S2). Figure 4 revealed that different tumor stages showed heterogeneous distribution of the cellular network entropy. For BRCA, COAD, and LUAD, stage IV had a lower genome-wide local network entropy compared to that of stages I $(p<0.01$, Wilcoxon rank-sum test), II $(p<0.01)$ and III $(p<0.01)$. In contrast, stage IV in HNSC had a higher genome-wide local network entropy compared to that in its stages I $\left(p<2.2 \times 10^{-16}\right)$, II $(p<0.01)$ and III $(p<0.01)$. THCA and KIRC only showed a minor genome-wide local network entropy changes across 4 different stages. Low network entropy observed in stage IV (metastasis) compared to that of low stages (stages I to III) might be explained by tumor clonal evolution [24]. For example, during tumor subclonal evolution (e.g., a clonal sweep), a new clone that took over the entire population and replaced the ancestral clones would result in a homogeneous cell population 


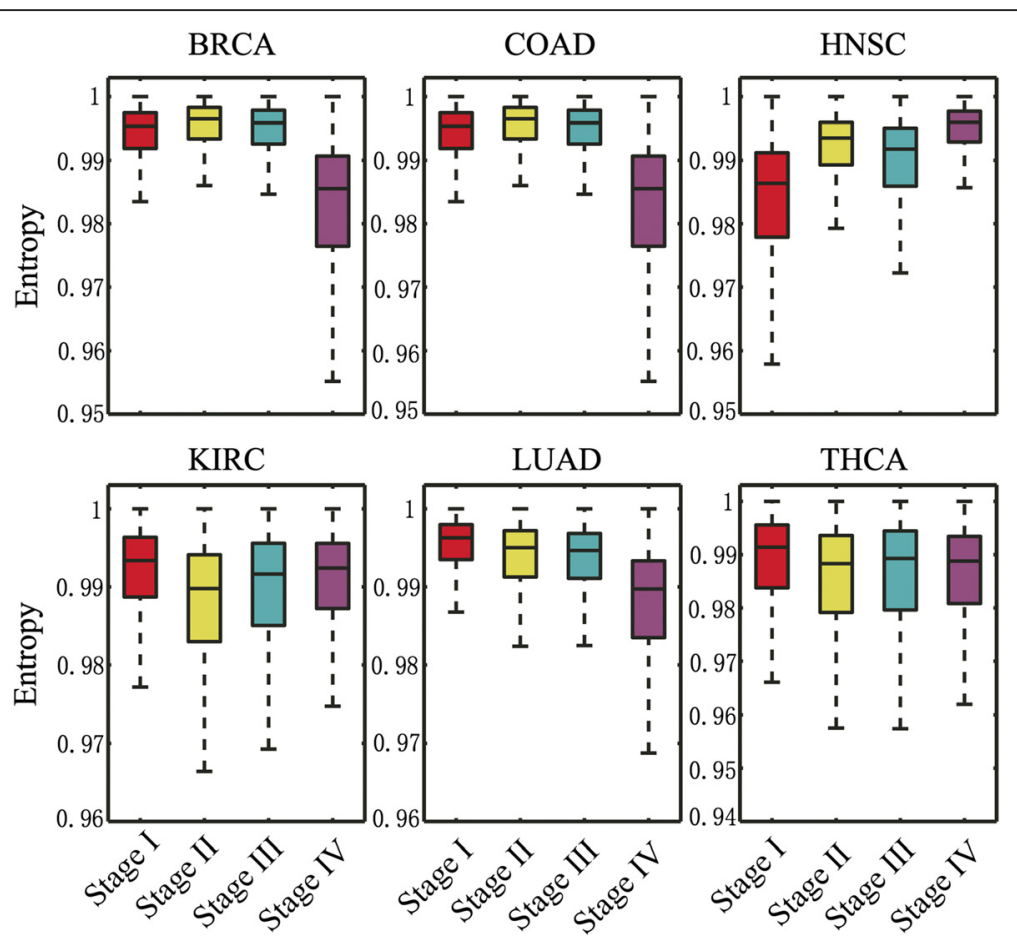

Fig. 4 Boxplots showing the distribution of genome-wide local network entropy across 4 stages (I-IV) of tumor progression in 6 cancer types. Abbreviations of 6 cancer types are provided in Fig. 1's legend

with low network entropy during tumor metastasis (e.g., breast cancer) $[24,25]$.

We next compiled a list of high-quality, significantly mutated genes (SMGs) in cancer from 4 pan-cancer genomic analysis projects, as described in our previous study [6]. A total of 614 SMGs were collected. Similar to the genome-wide network entropy analysis, BRCA and LUAD indicated the lowest network entropy for SMGs in stage IV $(p<0.01)$ compared to that in stages I-III (Additional file 1: Figure S1). HNSC had the highest network entropy for SMGs in stage IV compared to that in stages I-III $(p<$ 0.01). We next examined Cancer Gene Census (CGC) genes, which are well curated and have been widely used as a reference cancer gene set in various cancer-related studies [26, 27]. Similar trends were observed for CGC genes compared to that in SMGs and genome-wide analysis (Additional file 1: Figure S2). We further collected 477 oncogenes (OGs) and 1040 tumor suppressor genes (TSGs) from our previous study $[6,28]$, and then examined the network entropy value for OGs and TSGs. We found a similar network entropy distribution for OGs and TSGs compared to that for genome-wide genes, SMGs, and CGC genes across 4 stages in the 6 cancer types (Additional file 1: Figures S3 and S4). Taken together, our results suggested that different stages of tumor progression might be characterized by heterogeneous network entropy distribution for both genome-wide and cancerrelated genes: SMGs, CGC genes, OGs, and TSGs.

\section{Characterizing anticancer drug responses by network entropy}

We next investigated whether a subset of cancer cell lines showing different anticancer drug responses (e.g. sensitivity or resistance) could be characterized by dynamic network entropy. We compiled normalized microarray gene expression data and drug pharmacological data on cancer cell lines from the GDSC database $[15,16]$. We separated the cancer cell lines into two subsets (resistant and sensitive) based on drug maximal screening concentration described in two previous studies $[15,16]$. In order to reliably estimate covariance of two genes across a set of cancer cell lines, we selected cancer types that had at least 10 cell lines with drug responses and microarray gene expression data simultaneously. Based on this criterion, we compiled four molecularly targeted drugs (Gefitinib, Dasatinib, Nilotinib, and Temsirolimus) in 4 cancer types of cell lines (lung, breast, blood, and skin) from the GDSC database.

Figure 5 showed that 3 tyrosine kinase inhibitors (Gefitinib, Dasatinib, and Nilotinib) resistant cell lines had a higher genome-wide local network entropy compared to that of their sensitive cell lines in blood and lung cancer $\left(p<2.2 \times 10^{-16}\right.$, Wilcoxon rank-sum test). However, serine/ threonine protein kinase inhibitor (Temsirolimus) resistant cell lines had a lower genome-wide local network entropy compared to that of the sensitive cell lines in all of the 4 cancer types: lung, breast, blood, and skin cancer, as shown 


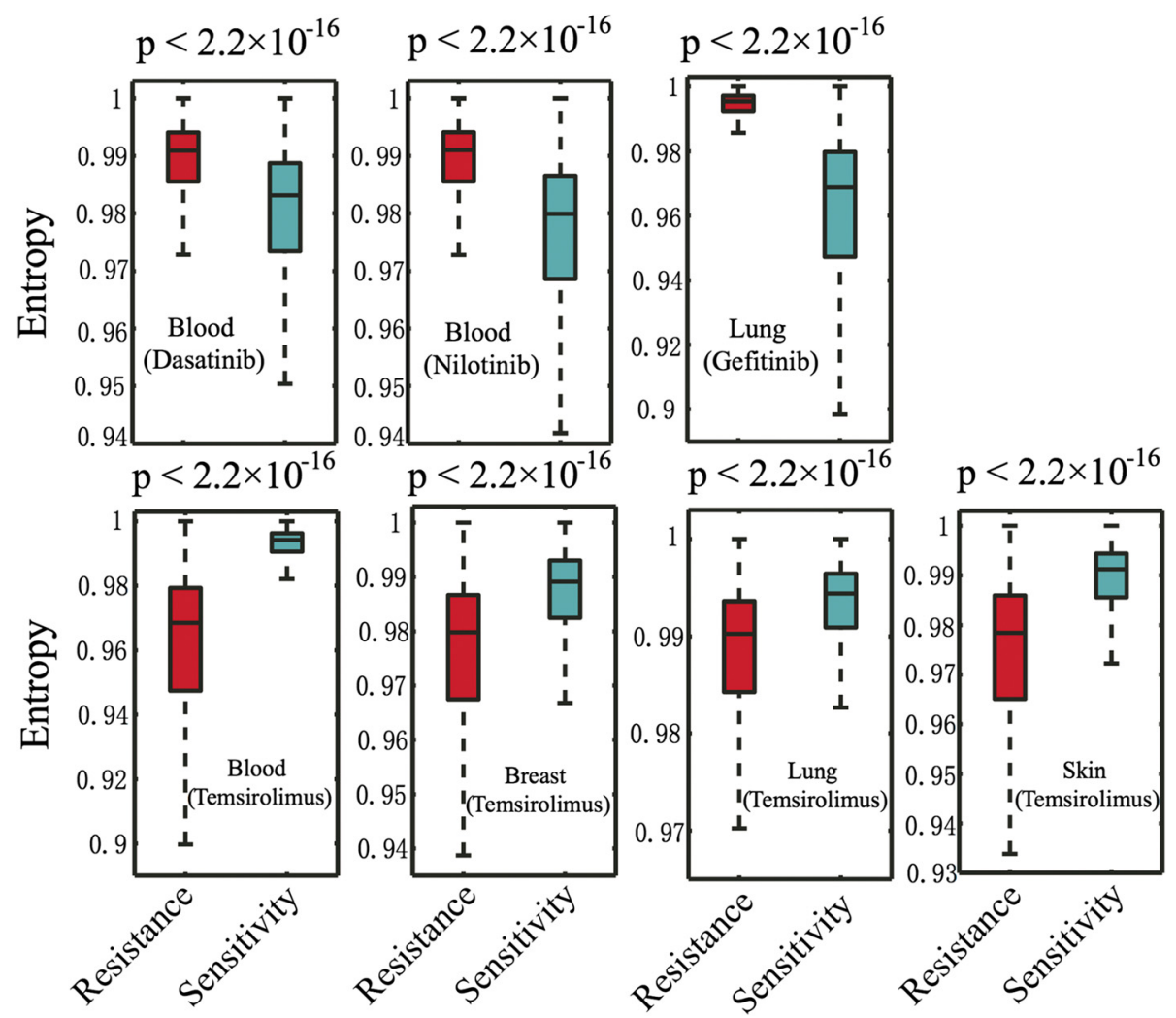

Fig. 5 Boxplots showing the distribution of genome-wide local network entropy between drug resistant and sensitive cancer cell lines for 4 targeted anticancer drugs in 4 types of cancer: blood, lung, breast, and skin. Local network entropy distribution between drug resistant and sensitive cancer cell lines for 458 drug-sensitivity genes was provided in Additional file 1: Figure S5. The p-value was calculated by Wilcoxon rank-sum test

in Fig. 5. We further compiled 458 genes that were involved in sensitivity or resistance of 130 anticancer drugs from a previous study [16]. As shown in Additional file 1: Figure S5, similar network entropy distribution was observed for 458 drug-sensitivity genes compared to that in genome-wide local network entropy analysis (Fig. 5).

\section{Discussion}

\section{Smoking is associated with cancer cellular network} heterogeneity and drug responses

Previous studies revealed that some environmental factors like smoking were likely to increase heterogeneity within a tumor $[29,30]$. We examined the network entropy distribution in a set of tumor samples that included both smoking and non-smoking histories. Figure 3 revealed that smokers had higher genome-wide network entropy compared to that of non-smokers in all of the 3 smoking-related cancer types: LUAD, LUSC, and HNSC. In our recent studies, we also found that smokers had unique mutation signatures [31] and higher mutational heterogeneity using a mathematical model [6]. Collectively, environmental factors, such as smoking, may play crucial roles during tumorigenesis and often speedup cancer cellular network heterogeneity. Furthermore, the increased cancer cellular network heterogeneity caused by smoking may be associated with drug responses. For example, Fig. 5 showed that Gefitinib resistant lung cancer cell lines were often characterized by elevated network entropy. Filosto et al. suggested that cigarette smoke mediates lung cancer development and resistance to tyrosine kinase inhibitors (e.g. Gefitinib) [32]. Kim et al. found that lung cancer patients with more than 30 pack-years smoking dosage have significantly poorer survival outcome on tyrosine kinase inhibitor therapy [33]. Collectively, this study suggested potential network-level evidence that smoking may increase cancer cellular network heterogeneity and further contribute to tyrosine kinase inhibitor resistance. Hence, our network analysis yielded a potential network-based predictor for quantitatively characterizing the clinical outcome of molecularly targeted treatment (e.g. tyrosine kinase inhibitor) in lung cancer.

\section{Limitations and future directions}

There are several potential limitations in current study. For example, we used large-scale RNA-Seq data across four stages of tumor progression in 6 cancer types from TCGA to study tumor progression quantified by network entropy. However, we did not observe a consistent 
pattern of network entropy distribution in four different stages of progression across 6 cancer types. One possible reason is that most of the current TCGA projects only generated RNA-Seq data for primary tumors. Although different tumor stage information was annotated for TCGA samples, most tumor samples sequenced in TCGA were collected from primary tumors, not metastatic tumors, limiting the accuracy of our network entropy analysis as shown in Fig. 4. Thus, further investigations are warranted for systematically examining the network entropy based on data sequenced in both primary and metastatic tumors, which we hope will be prompted by the findings herein. For example, a recent TCGA study sequenced 266 metastases and 67 primary cutaneous melanomas [34]. This will provide more useful datasets to examine melanoma cellular network heterogeneity in the future.

In addition, although we used a large-scale PIN for network entropy and unbalanced motif analysis, current network analyses also have some limitations, such as network incompleteness and possible data noise. For example, current PPI networks identified by highthroughput technologies may only cover less than $20 \%$ of all potential pairwise PPIs in the human cells [35, 36]. PPIs are tissue or cell type specificity. However, we assembled all PPIs from different tissues or cell types as a global background in this study, which may cause potential data bias. In addition, the unbalanced number in tumors, normal tissues, and different tumor stages (Additional file 1: Table S1) may also influence the results. For example, the number of stage IV BRCA samples was 15 , much less than the numbers of BRCA samples in stages I, II and III (Additional file 1: Table S2). While the number of stage IV LUAD sample (22) was smaller that that normal lung tissue samples (58), the network entropy in stage IV LUAD was higher than that of normal lung tissues $(p<0.01)$. This result suggested that the unbalanced number of tumor samples during tumor progression might not influence the overall conclusion in this study. Finally, ultra-mutated tumor samples in some cancer types such as colon cancer may also influence the analysis result. For instance, a small set of tumor samples can contribute to a large proportion (e.g., up to $40 \%$ ) of total somatic mutations observed in the whole cancer cohort [37]. Figure 4 revealed that stage IV had the lowest genome-wide network entropy distribution compared to that of stages I-III in COAD. However, we did not observe a similar trend of network entropy distribution for four cancer-related gene sets: SMGs, CGC genes, OGs, and TSGs, in COAD.

\section{Conclusion}

In this study, we proposed a network entropy and unbalanced motif approach to systematically investigate network features during tumor initiation, progression, and anticancer drug responses quantified by cellular network heterogeneity and modularity under the notion of Waddington's landscape. We found that tumorigenesis was characterized by increased network entropy and unbalanced motifs compared to that of normal tissues using TCGA data. Furthermore, the increased network entropy may be associated with anticancer drug resistance. In a case study, we found that smoking is characterized by the increased cellular network heterogeneity, suggesting potential network level evidence associated with tyrosine kinase inhibitor (e.g. Gefitinib) resistance induced by smoking in lung cancer. In addition, different stages of tumor progression are characterized by highly heterogeneous network entropy, which may contribute to high risk of drug resistance in the molecularly targeted cancer therapy. In summary, this study could provide new potential networkbased predictively prognostic and therapeutic biomarkers for cancer systems biology study and the molecularly targeted cancer therapeutics.

\section{Methods \\ Construction of protein interaction network}

We constructed a high-quality PIN covering 113,473 unique interactions connecting 13,579 protein-coding genes based on our previous studies [6, 13, 28, 38]. We implemented three data cleaning steps to select a highquality PPI pair. First, we only compiled a high-quality PPI pair if it was experimentally validated in human models through a well-defined experimental protocol. Second, we re-annotated all protein-coding genes using gene Entrez ID and the gene official symbols from the National Center for Biotechnology Information (NCBI) database [39]. Finally, self-loop interactions or duplicated PPI pairs were excluded. The detailed data collection and preparation are provided in our previous studies $[6,13,28]$.

\section{Preparation of RNA-Seq data and gene co-expression analysis}

We downloaded RNA-Seq V2 data from 3557 tumor samples across 8 cancer types and 418 matched normal tissues from TCGA (October 02, 2013) [40]. These 8 cancer types consisted of BRCA, COAD, HNSC, KIRC, LUAD, LUSC, THCA, and UCEC (Additional file 1: Table S1). In this study, we implemented two steps to define the genes that were expressed: (i) in a sample, we filtered out a gene whose mRNA expression was below the $20 \%$ of all mRNAs ordered by their expression level; and (ii) we further filtered out a gene that expressed in less than $20 \%$ of samples in the whole expression matrix. We also extracted RNA-Seq V2 data for smokers and never-smokers in LUAD, LUSC, and HNSC from TCGA (January 05, 2015) using the $R$ package 
implemented in TCGA-Assembler [41]. In addition, we collected normalized microarray gene expression data for drug sensitive versus resistant cancer cell lines from the GDSC database (July 01, 2014) [15, 16]. Finally, we calculated PCC value for each gene-gene pair and mapped PCC value of each gene-gene pair onto aforementioned PIN to construct CePINs for cancer types (including different stages of tumor progression), normal tissues, smokers versus non-smokers, and drug sensitive versus resistant cancer cell lines, respectively (Fig. 1a).

\section{Network entropy analysis}

In this study, we denoted $P C C_{i j}$ as PCC value of a gene coexpression pair between gene $i$ and $j$ in PIN. Since $-1 \leq$ $P C C_{i j} \leq 1$, the edge weights of CePIN can be redefined as $w_{i j}=\frac{1}{2}\left(1+P C C_{i j}\right)$ based on two previous studies $[9,12]$.

For each gene $i$, we calculate the local Shannon-Jayne entropy as follows:

$$
S_{i}=-\frac{1}{\log \left(k_{i}\right)} \sum_{j \in N(i)} p_{i j} \log \left(p_{i j}\right)
$$

Where $k_{i}$ is the number of gene i's neighbors, $N(i)$ is the set of gene $i$ 's neighbors in CePIN and $p_{i j}=\frac{w_{i j}}{\sum_{j \in N(i)} w_{i j}}$ is the proportion of gene $i$ 's total weights that links to gene $\mathrm{j}$.

We quantified cancer cellular network heterogeneity as a function of the network entropy under on the notion of Waddington's landscape [10, 17], which can be represented as the distribution of local entropies across the whole network.

\section{Balanced versus unbalanced motif analysis}

Relations between genes on CePIN often reflect a mixture of positive and negative PCC value (negative versus positive PPI in the right panel of Fig. 1a). The interplay between positive and negative relationships significantly affects the network structure. In this study, the signed triangle motifs on three genes are extracted from CePIN, where the edge $i j$ in the triangle motif is signed as positive when $P C C_{i j}>0$, and negative when $P C C_{i j}<0$. There are four types of signed triangle motifs (See Fig. 1c). Following the classical structural balance theory [42], the motifs with odd number ( 1 or 3 ) of positive edges are more plausible, which are considered as the balanced motifs (balanced motif Type I and Type II in Fig. 1c), while the motifs with even number (0 or 2$)$ of positive edges are considered as the unbalanced motifs (unbalanced motifs Type I and Type II in Fig. 1c). And the balanced motifs should be more prevalent in stable systems.
Here, the proportion of the unbalanced motifs $\left(p_{u m}\right)$ could be used to represent the disorder level of the network structure:

$$
p_{u m}=\frac{N_{u m}}{N_{t m}}
$$

where, $N_{u m}$ is the number of the unbalanced motifs in the network, and $N_{t m}$ is the total number of the triangle motifs in the network. The large $p_{u m}$ value shows the more heterogeneous network structure, and we can detect the evolution of the various states for different cancer types by comparing the $p_{u m}$ value. To perform reliably balanced versus unbalanced motif analysis, we only kept the significantly co-expressed pairs having $\mathrm{p}$ value $<0.05$ (F-statistics) in each CePIN for unbalanced versus balanced motif analysis.

\section{Cancer gene sets}

We collected four cancer-related gene sets: 614 cancer SMGs, 487 CGC genes, 477 oncogenes, and 1040 TSGs, as briefly described in our previous study [28]. The abbreviations of these gene sets were described in the Results section. We further compiled 458 genes that were involved in sensitivity or resistance of 130 anticancer drugs from two previous studies $[13,16]$. In that study, the authors comprehensively identified drug-sensitivity genes on 639 human tumor cell lines using the integrated genomics analysis [16].

\section{Statistical analysis}

All statistical tests were performed using the $\mathrm{R}$ package (v3.0.1) [43].

\section{Additional file}

\begin{abstract}
Additional file 1: Table S1. The statistics of RNA-Seq data for tumors and normal tissues in 8 cancer types collected from TCGA. Table S2. The statistics of RNA-Seq data for four stages of tumor progression in 6 cancer types collected from TCGA. Figure S1. Local network entropy distribution for cancer significantly mutated genes among four stages (I-IV) of tumor progression in 6 cancer types. Figure S2. Local network entropy distribution for Cancer Gene Census (CGC) genes among four stages (I-IV) of tumor progression in 6 cancer types. Figure S3. Local network entropy distribution for oncogenes (OGS) among four stages (I-IV) of tumor progression in 6 cancer types. Figure S4. Local network entropy distribution for tumor suppressor genes (TSGS) among four stages (I-IV) of tumor progression in 6 cancer types. Figure S5. Local network entropy distribution for 458 drug-sensitivity genes in drug sensitive versus resistant cancer cell lines. (DOCX 994 kb)
\end{abstract}

\section{Acknowledgements}

We thank Chen-Ching Lin in Bioinformatics and Systems Medicine Laboratory for his valuable discussion and assistance on gene co-expression network analysis. This work was partially supported by National Institutes of Health grant (R01LM011177), The Robert J. Kleberg, Jr. and Helen C. Kleberg Foundation, and Ingram Professorship Funds. The funders had no role in study design, data collection and analysis, decision to publish, or preparation of the manuscript. 


\section{Declarations}

Publication of this article was charged from the faculty retention funds to Dr. Zhao from Vanderbilt University. This article has been published as part of BMC Systems Biology Volume 10 Supplement 3, 2016: Selected articles from the International Conference on Intelligent Biology and Medicine (ICIBM) 2015: systems biology. The full contents of the supplement are available online at http://bmcsystbiol.biomedcentral.com/articles/supplements/ volume-10-supplement-3.

\section{Availability of data and materials}

Datasets supporting the results of this article are also included in the additional files.

\section{Authors' contributions}

Z.Z. and F.C. conceived and designed the study. F.C. and C.L. carried out experiments and analyzed the data. B.S. interpreted the results. F.C. and Z.Z. interpreted the results and wrote the manuscript. All authors read and approved the final manuscript.

\section{Competing interests}

The authors declare that they have no competing interests.

\section{Consent for publication}

Not applicable

\section{Ethics approval and consent to participate}

Not applicable

\section{Author details}

${ }^{1}$ Department of Biomedical Informatics, Vanderbilt University School of Medicine, Nashville, TN, USA. ${ }^{2}$ Alibaba Research Center for Complexity Sciences, Hangzhou Normal University, Hangzhou, Zhejiang, China. ${ }^{3}$ Center for Systems Biology, Soochow University, Suzhou, China. ${ }^{4}$ Department of Cancer Biology, Vanderbilt University School of Medicine, Nashville, TN, USA. ${ }^{5}$ Department of Psychiatry, Vanderbilt University School of Medicine, Nashville, TN, USA. ${ }^{6}$ Center for Precision Health, School of Biomedical Informatics, The University of Texas Health Science Center at Houston, Houston, TX, USA

\section{Published: 26 August 2016}

\section{References}

1. Siegel R, Ma J, Zou Z, Jemal A. Cancer statistics, 2014. CA Cancer J Clin. 2014:64(1):9-29.

2. Mutation C, Pathway Analysis working group of the International Cancer Genome C. Pathway and network analysis of cancer genomes. Nat Methods. 2015;12(7):615-21.

3. Pe'er D, Hacohen N. Principles and strategies for developing network models in cancer. Cell. 2011;144(6):864-73.

4. Zhao J, Cheng F, Wang Y, Arteaga LC, Zhao Z. Systematic prioritization of druggable mutations in $\sim 5,000$ genomes across 16 cancer types using a structural genomics-based approach. Mol Cell Proteomics. 2016;15(2):642-56.

5. Zhang J, Lu K, Xiang Y, Islam M, Kotian S, Kais Z, Lee C, Arora M, Liu HW, Parvin $J \mathrm{D}$, et al. Weighted frequent gene co-expression network mining to identify genes involved in genome stability. PLoS Comput Biol. 2012;8(8):e1002656.

6. Cheng F, Liu C, Lin CC, Zhao J, Jia P, Li WH, Zhao Z. A gene gravity model for the evolution of cancer genomes: a study of 3,000 cancer genomes across 9 cancer types. PLoS Comput Biol. 2015;11(9):e1004497.

7. Creixell P, Schoof EM, Erler JT, Linding R. Navigating cancer network attractors for tumor-specific therapy. Nat Biotechnol. 2012;30(9):842-8.

8. Csermely P, Hodsagi J, Korcsmaros T, Modos D, Perez-Lopez AR, Szalay K Veres DV, Lenti K, Wu LY, Zhang XS. Cancer stem cells display extremely large evolvability: alternating plastic and rigid networks as a potential Mechanism: network models, novel therapeutic target strategies, and the contributions of hypoxia, inflammation and cellular senescence. Semin Cancer Biol. 2015:30:42-51.

9. West J, Bianconi G, Severini S, Teschendorff AE. Differential network entropy reveals cancer system hallmarks. Sci Rep. 2012;2:802.

10. Banerji CR, Severini S, Caldas C, Teschendorff AE. Intra-tumour signalling entropy determines clinical outcome in breast and lung cancer. PLoS Comput Biol. 2015;11(3):e1004115.
11. Newton PK, Mason J, Hurt B, Bethel K, Bazhenova L, Nieva J, Kuhn P. Entropy, complexity, and Markov diagrams for random walk cancer models. Sci Rep. 2014;4:7558

12. Teschendorff AE, Severini S. Increased entropy of signal transduction in the cancer metastasis phenotype. BMC Syst Biol. 2010;4:104.

13. Cheng F, Jia P, Wang Q, Zhao Z. Quantitative network mapping of the human kinome interactome reveals new clues for rational kinase inhibitor discovery and individualized cancer therapy. Oncotarget. 2014;5(11):3697-710.

14. Gillies RJ, Verduzco D, Gatenby RA. Evolutionary dynamics of carcinogenesis and why targeted therapy does not work. Nat Rev Cancer. 2012;12(7):487-93.

15. Yang W, Soares J, Greninger P, Edelman EJ, Lightfoot H, Forbes S, Bindal N, Beare D, Smith JA, Thompson IR, et al. Genomics of Drug Sensitivity in Cancer (GDSC): a resource for therapeutic biomarker discovery in cancer cells. Nucleic Acids Res. 2013;41:D955-61.

16. Garnett MJ, Edelman EJ, Heidorn SJ, Greenman CD, Dastur A, Lau KW, Greninger P, Thompson IR, Luo X, Soares J, et al. Systematic identification of genomic markers of drug sensitivity in cancer cells. Nature. 2012;483(7391):570-5.

17. Waddington $\mathrm{CH}$. The strategy of the genes: a discussion of some aspects of theoretical biology. London: Allen \& Unwin; 1957.

18. Stergachis AB, Neph S, Reynolds A, Humbert R, Miller B, Paige SL, Vernot B, Cheng JB, Thurman RE, Sandstrom R, et al. Developmental fate and cellular maturity encoded in human regulatory DNA landscapes. Cell. 2013;154(4): 888-903.

19. Polyak K. Heterogeneity in breast cancer. J Clin Invest. 2011;121(10):3786-8.

20. Govindan R, Ding L, Griffith M, Subramanian J, Dees ND, Kanchi KL, Maher CA, Fulton R, Fulton L, Wallis J, et al. Genomic landscape of non-small cell lung cancer in smokers and never-smokers. Cell. 2012;150(6):1121-34.

21. Facchetti $\mathrm{G}$, lacono G, Altafini C. Computing global structural balance in large-scale signed social networks. Proc Natl Acad Sci U S A. 2011; 108(52):20953-8.

22. Vinayagam A, Zirin J, Roesel C, Hu Y, Yilmazel B, Samsonova AA, Neumuller RA, Mohr SE, Perrimon N. Integrating protein-protein interaction networks with phenotypes reveals signs of interactions. Nat Methods. 2014;11(1):94-9.

23. Ma W, Trusina A, El-Samad H, Lim WA, Tang C. Defining network topologies that can achieve biochemical adaptation. Cell. 2009:138(4):760-73.

24. Klein CA. Selection and adaptation during metastatic cancer progression. Nature. 2013:501(7467):365-72.

25. Burrell RA, McGranahan N, Bartek J, Swanton C. The causes and consequences of genetic heterogeneity in cancer evolution. Nature. 2013: 501(7467):338-45.

26. Futreal PA, Coin L, Marshall M, Down T, Hubbard T, Wooster R, Rahman N, Stratton MR. A census of human cancer genes. Nat Rev Cancer. 2004;4(3): 177-83.

27. Jia P, Zhao Z. VarWalker: personalized mutation network analysis of putative cancer genes from next-generation sequencing data. PLoS Comput Biol. 2014:10(2):e1003460

28. Cheng F, Jia P, Wang Q, Lin CC, Li WH, Zhao Z. Studying tumorigenesis through network evolution and somatic mutational perturbations in the cancer interactome. Mol Biol Evol. 2014;31(8):2156-69.

29. Sprouffske K, Merlo LM, Gerrish PJ, Maley CC, Sniegowski PD. Cancer in light of experimental evolution. Curr Biol. 2012;22(17):R762-71.

30. McGranahan N, Burrell RA, Endesfelder D, Novelli MR, Swanton C. Cancer chromosomal instability: therapeutic and diagnostic challenges. EMBO Rep. 2012;13(6):528-38.

31. Jia P, Pao W, Zhao Z. Patterns and processes of somatic mutations in nine major cancers. BMC Med Genomics. 2014;7:11.

32. Filosto $\mathrm{S}$, Becker CR, Goldkorn T. Cigarette smoke induces aberrant EGF receptor activation that mediates lung cancer development and resistance to tyrosine kinase inhibitors. Mol Cancer Ther. 2012;11(4):795-804.

33. Kim MH, Kim HR, Cho BC, Bae MK, Kim EY, Lee CY, Lee JS, Kang DR, Kim JH. Impact of cigarette smoking on response to epidermal growth factor receptor (EGFR)-tyrosine kinase inhibitors in lung adenocarcinoma with activating EGFR mutations. Lung Cancer. 2014;84(2):196-202.

34. Cancer Genome Atlas Network, Electronic address imo, Cancer Genome Atlas N. Genomic classification of cutaneous melanoma. Cell. 2015;161(7):1681-96.

35. Stumpf MP, Thorne T, de Silva E, Stewart R, An HJ, Lappe M, Wiuf C. Estimating the size of the human interactome. Proc Natl Acad Sci U S A 2008;105(19):6959-64.

36. Hart GT, Ramani AK, Marcotte EM. How complete are current yeast and human protein-interaction networks? Genome Biol. 2006;7(11):120. 
37. Kandoth C, McLellan MD, Vandin F, Ye K, Niu B, Lu C, Xie M, Zhang Q, McMichael JF, Wyczalkowski MA, et al. Mutational landscape and significance across 12 major cancer types. Nature. 2013;502(7471):333-9.

38. Kim P, Cheng F, Zhao J, Zhao Z. ccmGDB: a database for cancer cell metabolism genes. Nucleic Acids Res. 2016;44(D1):D959-68.

39. Corrdinators NR. Database resources of the National Center for Biotechnology Information. Nucleic Acids Res. 2013;41:D8-20.

40. The Cancer Genome Atlas. http://cancergenome.nih.gov/. Accessed 01 Nov 2014.

41. Zhu Y, Qiu P, Ji Y. TCGA-assembler: open-source software for retrieving and processing TCGA data. Nat Methods. 2014;11(6):599-600.

42. Cartwright D, Harary F. Structural balance: a generalization of Heider's theory. Psychol Rev. 1956;63(5):277-93.

43. R package (v3.0.1). http://www.r-project.org/. Accessed 01 June 2014.

Submit your next manuscript to BioMed Central and we will help you at every step:

- We accept pre-submission inquiries

- Our selector tool helps you to find the most relevant journal

- We provide round the clock customer support

- Convenient online submission

- Thorough peer review

- Inclusion in PubMed and all major indexing services

- Maximum visibility for your research

Submit your manuscript at www.biomedcentral.com/submit
Biomed Central 